\title{
ON THE PARITY OF THE FOURIER COEFFICIENTS OF $j$-FUNCTION
}

\author{
M. RAM MURTY AND R. THANGADURAI \\ (Communicated by Ken Ono)
}

Abstract. Klein's modular $j$-function is defined to be

$$
j(z)=E_{4}^{3} / \Delta(z)=\frac{1}{q}+744+\sum_{n=1}^{\infty} c(n) q^{n}
$$

where $z \in \mathbb{C}$ with $\Im(z)>0, q=\exp (2 i \pi z), E_{4}(z)$ denotes the normalized Eisenstein series of weight 4 and $\Delta(z)$ is the Ramanujan's Delta function. In this short note, we show that for each integer $a \geq 1$, the interval $(a, 4 a(a+1))$ (respectively, the interval $\left.\left(16 a-1,(4 a+1)^{2}\right)\right)$ contains an integer $n$ with $n \equiv 7$ $(\bmod 8)$ such that $c(n)$ is odd (respectively, $c(n)$ is even).

\section{INTRODUCTION}

Let $z$ be a complex number with $\Im(z)>0$ and $q=e^{2 \pi i z}$. The modular invariant $j$-function defined as

$$
j(z)=\frac{E_{4}^{3}(z)}{\Delta(z)}
$$

where

$$
\Delta(z)=q \prod_{n=1}^{\infty}\left(1-q^{n}\right)^{24}
$$

is the Ramanujan's Delta function and

$$
E_{4}(z)=1+240 \sum_{n=1}^{\infty} \sigma_{3}(n) q^{n}
$$

is the normalized Eisenstein series of weight 4 . The Fourier expansion for $j(z)$ is

$$
j(z)=\frac{1}{q}+744+\sum_{n=1}^{\infty} c(n) q^{n}
$$

where $c(n)$ are integers.

It is well known that $c(n)$ is even whenever $n \not \equiv 7(\bmod 8)$. Indeed, a result of J. P. Serre implies that for almost all integers $n \not \equiv 7(\bmod 8)$, one has $c(n) \equiv 0$ $\left(\bmod 2^{t}\right)$ for any integer $t \geq 1$. Later, Ono and Taguchi 4 ] proved that for any

Received by the editors July 14, 2013.

2010 Mathematics Subject Classification. Primary 11F30; Secondary 11F03.

Key words and phrases. Fourier coefficients, modular forms, $j$-function. 
$t \geq 1$, there is a positive integer $\ell$ such that for every set of distinct odd primes $p_{1}, p_{2}, \cdots, p_{\ell}$, one has

$$
c\left(p_{1} p_{2} \cdots p_{\ell} m\right) \equiv 0 \quad\left(\bmod 2^{t}\right)
$$

whenever $m \geq 1$ is coprime to $p_{1} p_{2} \cdots p_{\ell}$ and $p_{1} p_{2} \cdots p_{\ell} m \not \equiv 7(\bmod 8)$. Also, recently, Ono and Ramsey [3], extending the work of Alfes [1], proved that for any $D \equiv 7(\bmod 8)$, there are infinitely many $n$ such that $c\left(D n^{2}\right)$ is even.

Regarding the odd parity of $c(n)$, using the $\bmod p$ analogue of Atkin-Lehner's theorem and using the generalized Borcherds product, Ono and Ramsey [3] proved that for any $D \equiv 7(\bmod 8)$, if there exists one odd integer $n$ such that $c\left(D n^{2}\right)$ is odd, then there are infinitely many odd integers $m$ such that $c\left(D m^{2}\right)$ is odd. In particular, it follows that there are infinitely many odd integers $m \equiv 7(\bmod 8)$ such that $c(m)$ is odd. This can be seen by taking $D=7$ and noting that $c(7)$ is odd.

In this short note, we shall prove the following theorems, in the spirit of O. Kolberg's [2] proof of parity of partition function. Moreover, the following theorems predict a range in which a suitable $n \equiv 7(\bmod 8)$ can be chosen such that $c(n)$ is odd (respectively, even). In particular, our theorem gives an elementary proof of the infinitude of $n$ 's with $n \equiv 7(\bmod 8)$ for which $c(n)$ is odd (respectively, even).

Theorem 1.1. For every $a \geq 1$, there exists an integer $n \in(a, 4 a(a+1)-1]$ with $n \equiv 7(\bmod 8)$, such that $c(n)$ is an odd integer. In particular, there are infinitely many odd integers $n \equiv 7(\bmod 8)$ for which $c(n)$ is an odd integer.

Note that when $a=1$ in Theorem 1.1 , we get that the interval $[1,7]$ contains an integer $n \equiv 7(\bmod 8)$ such that $c(n)$ is odd. This must be $n=7$. Indeed, $c(7)=44656994071935$, which is an odd integer.

Corollary 1.2. For all $x \geq 8$, we have

$$
\begin{aligned}
\{1 \leq n \leq x: c(n) \text { is odd }\} & =\{n \leq x: n \equiv 7(\bmod 8) \text { and } c(n) \text { is odd }\} \\
& \geq c_{0} \log \log x
\end{aligned}
$$

for some positive constant $c_{0}$.

Theorem 1.3. For all $a \geq 1$, there exists an integer $n \in\left[16 a-1,(4 a+1)^{2}-1\right]$ with $n \equiv 7(\bmod 8)$ such that $c(n)$ is even. In particular, there exist infinitely many integers $n \equiv 7(\bmod 8)$ for which $c(n)$ is even.

When $a=1$ in Theorem 1.3, we get that 15 and 23 lie in the interval $[15,24]$. Note that $c(15)$ and $c(23)$ are even integers.

Corollary 1.4. For all $x \geq 15$, we have

$$
\#\{1 \leq n \leq x: n \equiv 7 \quad(\bmod 8) \text { and } c(n) \text { is even }\} \geq c_{1} \log \log x
$$

for some positive constant $c_{1}$.

Corollary 1.5. For a given residue class $\epsilon(\bmod 2)$, there exist infinitely many $n$ such that $c(n) \equiv \epsilon(\bmod 2)$.

In their paper, Ono and Ramsey [3] mention that it is expected that for half of the $n \equiv 7(\bmod 8)$, we should have $c(n)$ odd. 


\section{Proofs of theorems and COROllaries}

We shall start with the following lemma.

Lemma 2.1. For all integer $n \geq 1$, we have

$$
\sum_{m \geq 0} c\left(n-(2 m+1)^{2}\right) \equiv 0 \quad(\bmod 2) .
$$

Proof. The well-known Jacobi identity says that

$$
\prod_{n=1}^{\infty}\left(1-q^{n}\right)^{3}=\sum_{k=0}^{\infty}(-1)^{k}(2 k+1) q^{k(k+1) / 2} .
$$

Since $(x+y)^{2^{m}} \equiv x^{2^{m}}+y^{2^{m}}(\bmod 2)$, we use $(2.2)$ in $(1.2)$ to write

$$
\Delta(z) \equiv q \prod_{n=1}^{\infty}\left(1-q^{8 n}\right)^{3} \equiv q \sum_{n=0}^{\infty} q^{8 n(n+1) / 2} \equiv \sum_{n=0}^{\infty} q^{(2 n+1)^{2}} \quad(\bmod 2) .
$$

By $(1.3)$, we have $E_{4}(z) \equiv 1(\bmod 2)$. Therefore, $(1.1)$ becomes

$$
j(z) \Delta(z) \equiv 1 \quad(\bmod 2) .
$$

From (1.4) and (2.3), we have

$$
j(z) \Delta(z) \equiv\left(\sum_{n=-1}^{\infty} c(n) q^{n}\right)\left(\sum_{n=0}^{\infty} q^{(2 n+1)^{2}}\right) \quad(\bmod 2) .
$$

Therefore, we get

$$
1 \equiv \sum_{n=0}^{\infty} \sum_{k \geq 0} c\left(n-(2 k+1)^{2}\right) q^{n} \quad(\bmod 2) .
$$

Now by comparing the coefficients of $q^{n}$ on both sides, we get the required congruence.

Proof of Theorem 1.1. Let $a \geq 1$ be a given integer. Assume that $c(m)$ is even for every $m \in(a, 4 a(a+1)-1]$. Put $n=4 a(a+1)$ in $(2.1)$. We get

$\sum_{k \geq 0} c\left(4 a(a+1)-(2 k+1)^{2}\right)=\sum_{k \geq 0} c(4 a(a+1)-4 k(k+1)-1) \equiv 0 \quad(\bmod 2)$.

In the above congruence, the term corresponding to $k=a$ is $c(-1)$ which is indeed 1 and hence $c(-1) \not \equiv 0(\bmod 2)$. When we put $k=a-j$, we get

$$
4 a(a+1)-4(a-j)(a-j+1)-1=8 j a-4 j^{2}+4 j-1=4 j(2 a-j+1)-1 .
$$

If we vary $j=1,2, \cdots, a-1$, then we see that

$$
4 j(2 a-j+1)-1 \geq 4(2 a-(a-1)+1)-1=4(a+2)-1>a
$$

for all $a \geq 1$. Therefore, if

$$
c(4 a(a+1)-4 k(k+1)-1) \text { are all even for all } k=1,2, \cdots, a-1
$$

and $k=a$, the above integer is odd. Therefore, their sum cannot be even, which is a contradiction. Hence there is an integer $n \in(a, 4 a(a+1)-1]$ for which $c(n)$ is an odd integer. 
Since

$$
j(z) \equiv \frac{1}{q \prod_{n=1}^{\infty}\left(1-q^{8 n}\right)^{3}} \equiv \sum_{k=-1}^{\infty} b(k) q^{8 k+7} \quad(\bmod 2),
$$

where $b(k) \equiv 0,1(\bmod 2)$, by comparing the Fourier coefficients on both sides, we get if $n \not \equiv 7(\bmod 8)$, we have $c(n) \equiv 0(\bmod 2)$ and if $c(n)$ is odd, then $n \equiv 7$ $(\bmod 8)$. Therefore the integer $n \in(a, 4 a(a+1)-1]$ (for which $c(n)$ is odd) must be an odd integer and $n \equiv 7(\bmod 8)$.

Proof Corollary 1.2. We want to count $n \leq x$ for which $c(n)$ is odd. For that we define $a_{0}=1, a_{1}=7$, for every $k \geq 2$

$$
a_{k}=4 a_{k-1}\left(a_{k-1}+1\right)-1=4 a_{k-1}^{2}+4 a_{k-1}-1 .
$$

Then, we partition the interval

$$
[1, x]=[1,7] \cup\left(7, a_{2}\right) \cup\left[a_{2}, a_{3}\right) \cup \cdots \cup\left[a_{\ell-1}, a_{\ell}\right) \cup\left[a_{\ell}, x\right]
$$

where $\ell$ is the largest integer $k$ such that $a_{k} \leq x$. By Theorem 1.1, we know each interval $\left[a_{k-1}, a_{k}\right]$ contains at least one integer $n \equiv 7(\bmod 8)$ for which $c(n)$ is odd. Hence, the number of $n \leq x$ with $n \equiv 7(\bmod 8)$ for which $c(n)$ is odd is at least $\ell$ and it is remains to find the value of $\ell$ as a function of $x$. Since

$$
a_{k}=4 a_{k-1}^{2}+4 a_{k-1}-1<8 a_{k-1}^{2} \text { for all } k \geq 0,
$$

we get

$$
a_{k} \leq 8^{k} a_{1}^{2^{k-1}} \leq 8^{2^{k}} \text { for all } k \geq 0
$$

Since $a_{\ell} \leq x$, we see that $\ell \geq c_{0} \log x$ which proves the corollary.

Proof of Theorem 1.3. For every $a \geq 1$, we denote the interval

$$
I_{a}:=\left[16 a-1,(4 a+1)^{2}-1\right] .
$$

We need to prove that $I_{a}$ contains an integer $n \equiv 7(\bmod 8)$ for which $c(n)$ is even.

Suppose we assume that $c(n)$ is odd for every integer $n \equiv 7(\bmod 8)$ and $n$ lies in the interval $I_{a}$. Put $n=(4 a+1)^{2}-1$ in $(2.1)$ and we get

$$
\sum_{k \geq 0} c\left((4 a+1)^{2}-1-(2 k+1)^{2}\right) \equiv 0 \quad(\bmod 2)
$$

Note that the argument of $c$ in the summands is $(4 a+1)^{2}-1-(2 k+1)^{2} \equiv-1$ $(\bmod 8)$ and $(4 a+1)^{2}-1-(2 k+1)^{2} \in I_{a}$ for all $k=0,1, \cdots, 2 a-1$. When we put $j=2 a$, we get $c(-1)$ which is an odd integer. By assumption, we get $2 a$ number of 1 's and $c(-1)$ add up to $0(\bmod 2)$, which is a contradiction as $c(-1)$ is odd. Thus, there exists $n \in I_{a}$ with $n \equiv 7(\bmod 8)$ such that $c(n)$ is an even integer.

Proof Corollary 1.4. We want to count $n \leq x$ with $n \equiv 7(\bmod 8)$ for which $c(n)$ is even. Since we know $c(15)$ and $c(23)$ are even integers, we define $a_{0}=1, a_{1}=15$, for every $k \geq 2$ as

$$
a_{k}=\left(4 a_{k-1}+1\right)^{2}-1
$$

Then, we see that the disjoint union of the following intervals

$$
[1,15] \cup(15,25) \cup\left[a_{1}, a_{2}\right) \cup \cdots \cup\left[a_{\ell-1}, a_{\ell}\right) \cup\left[a_{\ell}, x\right] \subset[1, x]
$$

where $\ell$ is the largest integer $k$ such that $a_{k} \leq x$. By Theorem 1.3, we know each interval $\left[a_{k-1}, a_{k}\right]$ contains at least one integer $n \equiv 7(\bmod 8)$ for which $c(n)$ is 
even. Hence, the number of $n \leq x$ and $n \equiv 7(\bmod 8)$ for which $c(n)$ is even is at least $\ell$. Since $a_{k} \leq 32 a_{k-1}^{2}$ for all $k \geq 0$, we get,

$$
a_{k} \leq 32^{k} a_{1}^{2^{k-1}} \leq 32^{2^{k}} \text { for all } k \geq 0
$$

and hence we get the result.

\section{ACKNOWLEDGEMENTS}

This work was done while both the authors were visiting Kerala School of Mathematics (KSOM), Kozhikode and the Institute of Mathematical Sciences, Chennai. The authors are grateful to both the institutes for their hospitality during their visit.

\section{REFERENCES}

[1] Claudia Alfes, Parity of the coefficients of Klein's j-function, Proc. Amer. Math. Soc. 141 (2013), no. 1, 123-130, DOI 10.1090/S0002-9939-2012-11502-3. MR2988716

[2] O. Kolberg, Note on the parity of the partition function, Math. Scand. 7 (1959), 377-378. MR.0117213 (22 \#7995)

[3] Ken Ono and Nick Ramsey, A mod $\ell$ Atkin-Lehner theorem and applications, Arch. Math. (Basel) 98 (2012), no. 1, 25-36, DOI 10.1007/s00013-011-0347-x. MR2885530

[4] Ken Ono and Yuichiro Taguchi, 2-adic properties of certain modular forms and their applications to arithmetic functions, Int. J. Number Theory 1 (2005), no. 1, 75-101, DOI 10.1142/S1793042105000066. MR2172333(2006e:11057)

Department of Mathematics, Queen's University, Kingston, Ontario, Canada, K7L $3 \mathrm{~N} 6$.

E-mail address: murty@mast.queensu.ca

Harish-Chandra Research Institute, Chhatnag Road, Jhunsi, Allahabad 211019, India

E-mail address: thanga@hri.res.in 